Monatsschr Kinderheilkd 2015 • 163:671-672

DOI 10.1007/s00112-014-3238-y

Online publiziert: 19. Juni 2015

๑) Springer-Verlag Berlin Heidelberg 2015

S. Wirth ${ }^{1} \cdot$ F. Zepp ${ }^{2}$

${ }^{1}$ Zentrum für Kinder- und Jugendmedizin, HELIOS Klinikum Wuppertal, Universität Witten/Herdecke, Wuppertal

${ }^{2}$ Klinik für Kinder- und Jugendliche, Universitätsmedizin Mainz

\title{
Kinder- und Jugendpsychiatrie
}

Die Basiserhebung „Studie zur Gesundheit von Kindern und Jugendlichen in Deutschland“ (KiGGS) bei insgesamt 17.641 Kindern und Jugendlichen im Alter bis 17 Jahre machte deutlich, dass es in den letzten Jahrzehnten zunehmend zu einer Verschiebung von somatischen zu psychischen Auffälligkeiten gekommen ist. Im Hinblick auf emotionale und Verhaltensauffälligkeiten sowie psychische Störungen kann davon ausgegangen werden, dass ca. 15\% der Kinder und Jugendlichen im Alter zwischen 3 und 17 Jahren einer Risikogruppe angehören. Bei 5\% wurde ein Aufmerksamkeitsdefizit- und Hyperaktivitätssyndrom diagnostiziert, und bei 20\% der Jugendlichen im Alter zwischen 11 und 17 Jahren lagen Symptome einer Essstörung vor [1]. Die Ursachen sind sicher vielfältig und teilweise auch durch einen veränderten Lebensstil mit einerseits hohen Ansprüchen und andererseits großen Freiheiten und Möglichkeiten erklärbar.

\section{) Zirka 15\% der 3- bis 17-Jährigen gehören einer Risikogruppe an}

In den 5 Beiträgen zum Leitthema „Kinder- und Jugendpsychiatrie“ berichten namhafte Autoren aus der Kinder- und Jugendpsychiatrie über klinisch bedeutende und zunehmend häufiger diagnostizierte Krankheitsentitäten.

Im 1. Beitrag stellt Sinzig, Bonn, aktuelle Aspekte zu Autismus-Spektrum-Störungen vor. Die Diagnose wird in den letzten Jahren häufiger gestellt, was mit einer gesteigerten Aufmerksamkeit hinsichtlich des Krankheitsbilds und der Einführung einer standardisierten diagnostischen Vorgehensweise korreliert. Dies ist von großer Bedeutung, da eine frühe Diagnosestellung und korrekte Einordnung die frühzeitige Einleitung von adäquaten Hilfsangeboten sowie Interventionen ermöglichen und damit die Weiterentwicklung der Betroffenen maßgeblich beeinflussen.

Das 2. Thema befasst sich mit Zwangsstörungen im Kindes- und Jugendalter. Mit einer Prävalenz von 1-2\% handelt es sich um eine durchaus häufige, die Lebensführung sehr beeinträchtigende psychische Erkrankung, die rechtzeitig diagnostiziert werden muss, um eine Desozialisierung zu vermeiden. Wewetzer, Köln, beschreibt die aktuellen Behandlungsstrategien und macht deutlich, dass die multimodal ausgerichtete Therapie sehr individuell auf den Patienten angepasst werden muss.

Es folgen 2 lesenswerte Beiträge zu Essstörungen in Kindheit und Adoleszenz (Herpertz-Dahlmann u. Hagenah) sowie spezielle bei Jugendlichen mit Diabetes mellitus (Geisbüsch u. Bühren) aus der Arbeitsgruppe der Kinder und Jugendpsychiatrie in Aachen. Allgemein nehmen Essstörungen in unserer Alltagswahrnehmung zu, und Fachleute gehen davon aus, dass bis zu 10\% aller jugendlichen Mädchen in irgendeiner Form betroffen sind. Anorexia nervosa ist die Essstörung mit der höchsten Mortalität und allgemein schwersten Beeinträchtigung. Der Übersichtsbeitrag von Herpertz-Dahlmann et al. geht detailliert auf weitere Störungen wie „binge eating“, Bulimia nervosa und Störungen mit Vermeidung oder Einschränkung der Nahrungsaufnahme ein. Bei die- 
sen Störungsbildern, die häufig nur stationär suffizient behandelt werden können, ist eine frühe Diagnose und fachlich kompetente Einordnung notwendig. Dies umso mehr, da Patient und Umgebung häufig verdrängend reagieren. Interessant ist die Tatsache, dass offensichtlich Störungen des Essverhaltens bei Kindern und Jugendlichen mit Typ-1-Diabetes deutlich häufiger sind als in der Normalbevölkerung. Diese Patientengruppe muss daher unter diesem Aspekt besonders betreut werden.

Rehbein et al., Hannover, beschäftigen sich mit der Computerspielabhängigkeit im Jugendalter. Dies ist zweifelsfrei ein hochaktuelles Thema, das den Erziehungsberechtigten und Pädagogen zunehmend Kopfzerbrechen bereitet. Die ,internet gaming disorder" geht für Betroffene mit geringerer Lebenszufriedenheit, wachsender Vereinsamung und damit auch erhöhter psychischer Belastung einher. Kinderund Jugendärzte sind gefragt, Risikofaktoren und besonders gefährdete Jugendliche rechtzeitig zu identifizieren.

Wir freuen uns über Ihr Interesse und wünschen allen Lesern einen für den Alltag nutzbringenden Erkenntnisgewinn.

\section{Korrespondenzadresse}

\section{Prof. Dr. S. Wirth}

Zentrum für Kinder- und Jugendmedizin HELIOS Klinikum Wuppertal, Universität Witten/Herdecke Wuppertal

stefan.wirth@helios-kliniken.de

Interessenkonflikt. S. Wirth und F. Zepp geben an, dass kein Interessenkonflikt besteht

\section{Literatur}

1. Hölling H, Schlack R, Kamtsiuris $P$ et al (2012) Die KiGGS-Studie. Bundesgesundheitsblatt Gesundheitsforschung Gesundheitsschutz 55:836-842

\section{Supen log, 5}

Prof. Dr. S. Wirth

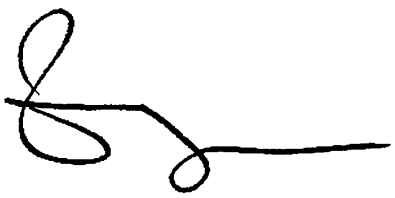

Prof. Dr. F. Zepp

\section{Kindesmisshandlung}

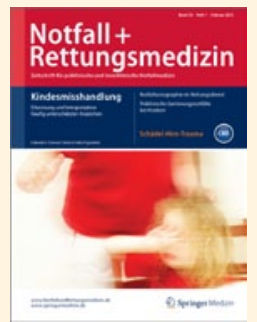

Jedes Jahr werden in Deutschland über 200.000 Kinder körperlich und seelisch misshandelt. Jede Wochen sterben 3 Kinder an den Folgen körperlicher Gewalt.

Diese Gewalt geschieht in jedem Milieu, ob Plattenbau, Reihenhaus oder Villa. Die derzeitige Zahl und Schwere der Kindesmisshandlungen ist inakzeptabel und ihre Verringerung muss dringend als gesamtgesellschaftliche Aufgabe wahrgenommen werden. Besonders wichtig dabei ist es, erste Anzeichen von Gewalt gegen Kinder im Alltag, in Praxis und Klinik zu erkennen und entsprechend zu handeln.

Lesen Sie im Schwerpunktheft „Kindesmisshandlung" (Ausgabe 1/2015) der Notfall+Rettungsmedizin, wann ein Verdacht gerechtfertigt ist und wie Sie damit umgehen:

- Verdacht auf Kindesmisshandlung

- Diagnostische Kriterien der Hautverletzungen bei Kindesmisshandlung

- Tödliches Schütteltrauma

- Das Recht zur Weitergabe von Informationen bei Gefährdung des Kindeswohls

Bestellen Sie diese Ausgabe zum Preis von 38,- EUR zzgl. Versandkosten bei Springer Customer Service Center Kundenservice Zeitschriften Haberstr. 7

69126 Heidelberg

Tel.: +49 6221-345-4303

Fax: +49 6221-345-4229

E-Mail: leserservice@springer.com

Suchen Sie noch mehr zum Thema? Mit e.Med, dem Online-Paket von Springer Medizin, können Sie schnell und komfortabel in über 500 medizinischen Fachzeitschriften recherchieren.

Weitere Infos unter springermedizin.de/eMed. 